\title{
Schreiben im Kollektiv von Händen und Dingen
}

Goethes Schreibkalendervon 1822/23

\author{
Cornelia Ortlieb
}

Schreiben ist eine einsame Tätigkeit. Die je spezielle Interaktion von Hirn, Auge und Hand ist weitgehend uneinsehbar und als Praktik trotz aller Beobachtungsmöglichkeiten in mehr oder weniger expliziten Laborsituationen letztlich nur in ihren Spuren aufzufinden, die selbstredend einer je speziellen Lektüre und Interpretation bedürfen. ${ }^{1}$ Wird in alter Tradition dabei nach wie vor der oder die Schreibende ins Zentrum der Aufmerksamkeit gerückt, kann übersehen werden, in welchem Maß nicht-menschliche Akteure oder Aktanten an Schreibprozessen beteiligt sind, sie formieren, regulieren oder sogar determinieren können. ${ }^{2}$ Mehr noch, die Grenze zwischen den andernorts klar unterschiedenen Personen und Dingen kann gerade in dieser geteilten Arbeit immer nur vorläufig gezogen werden, wenn etwa das Schreiben auf einer Tastatur gleichermaßen in den tippenden Fingern, den niedergedrückten Kunststofftasten, den unsichtbaren Befehlen und Codes im Hintergrund der Hardware und in den Textverarbeitungsprogrammen oder schließlich in den wie selbsttätig auf einem Bildschirm als Lichteffekte erscheinenden Buchstaben und Satzzeichen verortet werden kann. Die Physikerin und Philosophin Karen Barad, die für einen neuen „agentiellen Realismus“ eintritt, wählt das ähnliche Beispiel des Ausdruckens von Texten am Computer, um die Verschiebung der Wirk- und Handlungsmacht von der unterstellten Intention des menschlichen Akteurs zu den verschiedenen Maschinen zu beschreiben und die nicht zu beantwortende Frage zu stellen, wer denn nun eigentlich ,gedruckt' habe, die Hand an der Computermaus, der Computer, der Drucker oder alle zusammen? ${ }^{3}$

1 Solche Einzeluntersuchungen und ihre methodische Reflexion versammelt die Schriftenreihe Martin Stingelin (Hrsg.), Zur Genealogie des Schreibens, München: Fink 2oo4ff.

2 Vgl. für die Wissenschaftsgeschichte und -forschung als Geschichte von Praktiken die entsprechenden Arbeiten Bruno Latours, für die Unterscheidung von Akteuren und Aktanten etwa Bruno Latour, Wir sind nie modern gewesen. Versuch einer symmetrischen Anthropologie, dt. von Gustav Roßler, Frankfurt/Main: Suhrkamp 2008, für die einvernehmliche stille Zusammenarbeit von Menschen und Dingen Bruno Latour, „Der Berliner Schlüssel“, in: ders., Der Berliner Schlüssel. Erkundungen eines Liebhabers der Wissenschaften, dt. von Gustav Roßler, Frankfurt/Main: Suhrkamp 1996, S. 37-52.

3 Karen Barad, Agentieller Realismus. Über die Bedeutung materiell-diskursiver Praktiken, dt. von Jürgen Schröder, Berlin: Suhrkamp 2012, S. 14ff. 
Im Folgenden möchte ich zeigen, dass solche Schreib-Gemeinschaften auch lange vor der nun omnipräsenten Digitaltechnik immer bestanden haben und bestanden haben müssen, denn das Schreiben ist per definitionem auf Materialien und Werkzeuge angewiesen und geschieht somit niemals im luftleeren Raum reiner Geisttätigkeit, auch wenn eine lange westliche Tradition diese materiellen Anteile einer idealistisch überhöhten Praxis des Schreibens oder Dichtens häufig unterbetont oder getilgt hat. ${ }^{4}$ Es ist also trivial, das Zusammenwirken von Schreibenden, Schreibmitteln und Schriftträgern als unhintergehbare Voraussetzung jeder „Schreibszene“ zu konstatieren, benennt diese vielzitierte Formel nach Rüdiger Campe doch genau solch ein "nichtstabiles Ensemble von Sprache, Instrumentalität und Geste“..5 Und Martin Stingelin hat im Anschluss an Campe betont, dass sich je verschiedene Begriffe ,des' Schreibens ergeben, wenn der Anteil der „Idee“ oder der des „Werkzeugs“ privilegiert wird. ${ }^{6}$ Stingelin erläutert diesen Umstand anhand der Unterscheidung der zwei Schreibenden-Prototypen des ,Begeisterten' und des,Kalligraphen': Ersterer ist „von einer zu schreibenden Botschaft, einer Idee erfüllt“ und mag sich „bei seiner ,Be-Geisterung“" nicht vom „Automatismus“ regelgeleiteten Schreibens oder der "Mechanik“ von Werkzeug-Handhabungen ablenken lassen, letzterem „bedeuten“ die vermeintlich „äußerlichsten Anzeichen des Schreibens den innigsten Wert", und er richtet sein ganzes Augenmerk auf das „Zusammenspiel von Oberfläche, Werkzeug und Zeichen“.7 Allerdings fehlt es, trotz vieler detailgenauer Untersuchungen der Schreib(prozess)forschung, noch an Arbeiten, die solche Kollektive, Ensembles oder stille Gemeinschaften von Schreibenden und Dingen in Fallstudien konturieren könnten. Dafür mit Johann Wolfgang Goethe einen der

4 So ist in programmatischen Texten des 19. Jahrhunderts, besonders prominent in Hegels Ästhetik, das Schreiben in diesem emphatischen Sinn eine Tätigkeit, die keine Medien und Materialien kennt, und Poesie die „absolute wahrhafte Kunst des Geistes und seiner Äußerung als Geist“ (Georg Friedrich Wilhelm Hegel, Werke in zwanzig Bänden, Bd. 15: Ästhetik III, hrsg. von Eva Moldenhauer und Karl Markus Michel, Frankfurt/Main: Suhrkamp 1970, S. 224). Vgl. zu Visionen eines solchen nicht-handwerklichen Schreibens in der europäischen Literatur seit der Romantik Cornelia Ortlieb, „Zwischen Kopf und Papier. Phantasmen des Schreibens ohne Hände von Coleridge bis Bernhard“, in: Sprache und Literatur 43 (2012), H. 110, S. 22-37.

5 Rüdiger Campe, „Die Schreibszene, Schreiben“, in: Hans Ulrich Gumbrecht und Karl Ludwig Pfeiffer (Hrsg.), Paradoxien, Dissonanzen, Zusammenbrüche. Situationen offener Epistemologie, Frankfurt/Main: Suhrkamp 1991, S. 759-772, hier S. 76o.

6 Martin Stingelin, „,Schreiben'. Einleitung“, in: ders. (Hrsg.), „Mir ekelt vor diesem tintenklecksenden Säkulum“. Schreibszenen im Zeitalter der Manuskripte, München: Fink 2004, S. 7-21, hier S. 13 f.

7 Ebd., S. 14, mit Zitat von Sabine Mainberger, Schriftskepsis. Von Philosophen, Mönchen, Buchhaltern, Kalligraphen, München: Fink 1995, bes. S. 132-195. 
prominentesten Schreibenden der deutschen Literatur- und Kulturgeschichte zu wählen, liegt schon deshalb nahe, weil er in einem langen Leben und überbordenden Werk genügend Beispiele für den ersten wie für den zweiten Typus des Schreibenden bieten kann, abgesehen davon, dass er als späterer Schreib-Verweigerer mit seiner konsequenten Wendung zum Diktat und anderen Formen der nicht-eigenhändigen Aufzeichnung - wie etwa in den Gesprächen, die Johann Peter Eckermann vorgeblich treu notiert hat - bei gleichbleibend unvergleichlich hoher Produktivität auch interessante Sonderfälle dieser eigentlich alltäglichen Praktik erkennen lässt. Entsprechend sind seine Schreibarbeiten aus späten Jahren für eine solche Untersuchung besonders aufschlussreich, weil sie mit der seltenen Rückkehr zur eigenhändigen Niederschrift auch andere Gewichtungen der Bedeutung bestimmter Aufzeichnungen erahnen lassen, wie am Beispiel der Schreibkalender von 1823 gezeigt werden kann.

Dabei handelt es sich gewissermaßen um ein eigenes kleines Kollektiv: Die im Titel meines Beitrags noch unentschiedene Zahl lässt sich historisch präzisieren, wenn einerseits die Datierung der massenhaft produzierten und kommerziell vertriebenen Weimarer Taschenkalender berücksichtigt wird und andererseits deren Verwendung und entsprechende Umdeutung durch Goethe. Denn bei der berühmten letzten Böhmen-Reise von 1823 hat Goethe nicht nur den Kalender des Vorjahrs 1822 mitgeführt, den er und andere als Notizbuch für diverse Aufzeichnungen genutzt haben, sondern auch den noch aktuellen Kalender von 1823, der gleichfalls mit Notizen unterschiedlicher Art von verschiedenen Händen gefüllt ist. ${ }^{8}$ Findet der erste, ältere Kalender seit einigen Jahren schon deshalb besonderes Interesse, weil er die ersten Entwürfe des großen Gedichts enthält, das unter dem Gattungstitel Elegie mit dem Zusatz von Marienbad berühmt geworden ist, so liegt der zweite weitgehend

8 Den Kalender von 1822 zitiere ich der Zugänglichkeit halber nach seiner ersten Faksimileausgabe, Johann Wolfgang Goethe, Elegie von Marienbad. Faksimile einer Urschrift. September 1823, hrsg. von Christoph Michel und Jürgen Behrens, Frankfurt/Main: Insel 1983. Vgl. zu den Implikationen dieser Aufbereitung Cornelia Ortlieb, „Das Artefakt der Dichtung. ,Goethe's Schreib-Calender 1822“', in: Ulrike Gleixner et al. (Hrsg.), Biographien des Buches, Göttingen: Wallstein 2017, S. 228-249. Im Rahmen der Weimarer Ausstellung „Weimarer Klassik - Kultur des Sinnlichen“ ist der Taschenkalender nochmals als Faksimile in mehreren Exemplaren reproduziert und an einer der sogenannten „hands on“-Stationen zur freien Handhabung ausgelegt gewesen, vgl. auch Cornelia Ortlieb, „Schreib=Calender für das Jahr 1822", in: Sebastian Böhmer et al. (Hrsg.), Weimarer Klassik. Kultur des Sinnlichen. Katalog, Berlin, München: Deutscher Kunstverlag 2012, S. 318. Der Kalender von 1823 liegt im Weimarer Goethe- und Schiller-Archiv, Johann Wolfgang Goethe: Gedruckter Weimarischer Kalender aus dem Jahre 1823 mit Gedicht- und Briefentwürfen und Notizen, 1823, 42 Blatt, GSA $27 / 68$, S. 4 O. 
unbeachtet im Archiv, obgleich auch er Teil des komplexen Schreibprozesses von Marienbader Gedichtversen ist. Mit Blick auf diese gleichermaßen alltäglichen und einzigartigen Schriftträger, ihre Rolle und Funktion und die Wechselwirkung von Aufzeichnungsmaterialien und Schreibspur, wird es somit auch darum gehen, solche Ensembles zu konturieren und dabei bestimmte Grundannahmen philologischer Lektüren zu reflektieren, etwa die häufig stillschweigend unterstellten teleologischen Ordnungen, in denen sich diverse Schriftgebilde zu ,Stufen` eines allmählich zu vollendenden ,Textes‘ fügen sollen.

\section{I.}

Bereits um 1800 ermöglicht der Bleistift als neues Schreibwerkzeug ein Schreiben in Lebenslagen und Situationen, in denen die umständliche Handhabung von Feder und Tinte nicht möglich ist, etwa unterwegs, im Freien, auf wenig adäquaten Unterlagen. ${ }^{9}$ Goethe hat etwa in einer vielzitierten Hommage an diese neue Beweglichkeit und Unmittelbarkeit sein eruptives Dichten in einem Zug geschildert, das ihm nur mit Bleistift möglich war. ${ }^{10}$ Entsprechend mag die wichtigste Funktion des Weimarer Taschenkalenders in einer solchen permanenten Bereitstellung von Schreibpapier und passendem Werkzeug gelegen haben, und nur mit Bleistift konnten die Notizen des Taschenkalenders von 1822 ausgeführt werden. Denn seit dessen spektakulärer Wiederkehr nach etwa 150 Jahren in privatem, verborgenem Besitz sind durch zwei FaksimileEditionen die Bleistiftaufzeichnungen allgemein zugänglich geworden und unzweifelhaft als unterwegs angefertigte, offenbar in einer teils rüttelnden

Vgl. zur epochalen Zäsur durch die Einführung des neuen Schreibwerkzeugs Gerhard Kurz, „1795. Der Conté-Crayon. Auf dem Bleistiftweg oder die Erfindung eines Schreibgeräts", in: Roland Borgards, Almuth Hammer und Christiane Holm (Hrsg.), Kalender kleiner Innovationen. 50 Anfänge einer Moderne zwischen 1755 und 1856. Für Günter Oesterle, Würzburg: Königshausen \& Neumann 2006, S. 137-142; zur Geschichte seiner Herstellung Henry Petroski, Der Bleistift. Die Geschichte eines Gebrauchsgegenstands, dt. von Sabine Rochlitz, Basel u.a.: Birkhäuser 1995.

10 Goethe schildert das schnelle Aufschreiben als Mittel gegen das sofortige Vergessen seiner spontanen Einfälle und fährt fort: „In eben diesem Sinne griff ich weit lieber zu dem Bleistift, welcher williger die Züge hergab, denn es war mir einigemal begegnet, daß das Schnarren und Spritzen der Feder mich aus meinem nachtwandlerischen Dichten aufweckte, mich zerstreute und ein kleines Produkt in der Geburt erstickte." (Johann Wolfgang Goethe, Goethes Werke in 14 Bänden. Hamburger Ausgabe, hrsg. von Erich Trunz, Bd. 10: Dichtung und Wahrheit (1811-1830), München: Beck 1966, S. 8of.) Vgl. den einlässlichen Kommentar dieser Stelle in Stingelin, „,Schreiben“ (Anm. 6), S. 7 ff. 
Kutsche mit stoßweisen Erschütterungen schnell aufgezeichnete Entwürfe zu Gedichtversen identifiziert worden, die teils sofort oder mit kurzem Zeitabstand überarbeitet worden sind. Der Bleistift ist somit nicht nur das einzige dafür taugliche Instrument des Schreibens, sondern auch das Werkzeug der eigenhändigen Redaktion, einerseits der Spontankorrekturen, andererseits der offenbar späteren Anbringung einschlägiger Zeichen beim Wiederlesen, Abschreiben und Markieren des Verwerteten.

Dabei zeigen sich beim Aufschlagen des ungewöhnlich schmalen und hohen Büchleins, dem ein unbekannter Besitzer einen ledernen Schuber mit der Rückenprägung Goethe's Schreibcalender 1822 gegeben hat, schon im inneren Einband erste handschriftliche Eintragungen, die aber nicht von Goethe stammen, sondern von Dorothea Kuhn als Aufzeichnungen Johann Carl Stadelmanns identifiziert worden sind. Sie gelten geologischen und mineralogischen Gegenständen, über die sich der ausgebildete Buchdrucker selbstständig Kenntnisse angeeignet hat, so dass er als Goethes Diener in den Jahren 1814/15 und 1817-24 auch entsprechende Schreibarbeiten übernehmen konnte. ${ }^{11}$ Zunächst nach Art einer Liste mit abgegrenzten einzelnen Posten angelegt, sind die wenigen Worte dann offensichtlich aus Platzgründen in gebrochenen Zeilen angeordnet, die sich zwar nicht zu Versen fügen, aber doch eine auch poetische Reihung ergeben: „Kieselerden / Salzsaures Silber / Hornsilber / Niederschlag mit Lavendelöl / in der Glühhitze / doch ohne zu schmelzen / der Ränder / des Glases gleich / förmig aufgetra / gen gleichen Grad / der Hitze."12

Rechts gegenüber auf dem Vorsatzblatt sind Form und Gegenstand dieser Aufzeichnung übernommen, denn hier findet sich von Goethes Hand gleichfalls ein solches Hybrid aus Liste und Notiz in gebrochenen Zeilen: „Grüner Variolit / Sp[anischer] Andalusit. / Dienstag und Freytag / nicht / Mitw[och] und Sonnabend / am besten. // Ein charmantes / Stück von einem Manne. /

11 Vgl. [Christoph Michel und Jürgen Behrens,] „Erläuterungen zu den Notizen im SchreibKalender 1822", in: Goethe, Elegie von Marienbad (Anm. 8), S. 59-67, hier S. 59.

12 [Johann Carl Stadelmann, „Notizen“], in: Goethe, Elegie von Marienbad (Anm. 8), S. $16 f$. Hier und im Folgenden greife ich auch auf Formulierungen meines Aufsatzes über Goethes poetisches Abschreiben zurück, vgl. Cornelia Ortlieb, „Notieren, Billettieren, Übereignen. Goethes Dichten im Kopieren, Marienbad 1823“, in: Jörg Paulus, Andrea Hübener, Fabian Winter (Hrsg.), Duplikat, Abschrift und Kopie. Kulturtechniken der Vervielfältigung, Wien u.a.: Böhlau 202O, S. 131-154. Die Notizen im Kalender zitiere ich nach der Paginierung zu ihrer „Abbildung und Transkription“ im Kommentarband der ,großen“ Frankfurter Edition mit jeweils zwei Seitenangaben und übernehme der Übersichtlichkeit halber auch deren Titel: [Goethe,] „Die Urschrift K“, in: Goethe, Elegie von Marienbad (Anm. 8). Bemerkenswerterweise werden durch diese Titulierung auch die anderen Notizen von Goethes Hand zum Teil dieser „Urschrift“ des Gedichts. 
------ / Lotterie Loos. ${ }^{{ }^{13}}$ Die Anmerkung zu dieser Eintragung verzeichnet, was auf der Ablichtung der Seite gut zu erkennen ist: Die beiden Stein- und Mineralnamen und die gesamten Zeilen 1-6 sind eine „als erledigt durchgestrichene Vormerkung“, mit dafür typischen, auch von anderen Schreibenden regelmäßig genutzten diagonalen Strichen von links oben nach rechts unten, die das solchermaßen Durchkreuzte noch für die (Re-)Lektüre stehen lassen. ${ }^{14}$ Diese Notizen zu wissenschaftlichen Gesprächen, Verabredungen oder Erledigungen sind typischerweise nicht gemeint, wenn die Einträge im Kalender als „Urschrift K“ der Elegie bezeichnet werden; sie fehlen entsprechend in der Frankfurter Lese-Ausgabe, die auch die faksimilierten Seiten gekürzt um eben diesen Teil anbietet. ${ }^{15}$

Unabhängig von der Frage, inwieweit Stadelmann und andere Begleiter der Böhmen-Reise von 1823 mehr oder weniger indirekt zu den Versen der Elegie beigetragen haben, ist hier jedenfalls zuallererst ein kollektiver Schreibakt dokumentiert, an dem nicht nur die offensichtlich intentional arbeitenden Hände der beiden Schreibenden entscheidend beteiligt sind, sondern auch der Kalender als Gattung und Material, der mit seinen speziell formatierten Seiten zu eben solchen unspezifisch gemischten Einträgen einlädt oder auffordert und den dafür vorgesehenen Raum zugleich öffnet und entschieden begrenzt. Das zeigt sich eindrucksvoll beim Weiterblättern zum Anfang des Dichtens im emphatischen Sinn auf der Seite nach diesen beiden ersten Schreib-Arbeiten, links vom Titelblatt des Kalenders, den man allerdings um go Grad nach rechts drehen muss, um die quer eingeschriebenen Langzeilen mit ihren mehrfachen Sofort-Korrekturen, Streichungen und Überschreibungen entziffern zu können (Abb. 2.1). Die meist nicht eigens als solche ausgewiesene editorische Abschrift, ${ }^{16}$ die notwendigerweise mit der Vereindeutigung auch eine

13 Goethe, „Die Urschrift K“ (Anm. 12).

14 Michel/Behrens, „Erläuterungen“ (Anm. 11), S. 62. Vorgemerkt wurde demnach „das am 3. Oktober an Grüner abgesandte Mineralienkästchen“ (ebd.). Beide Einträge beziehen sich offensichtlich auf mineralogische Gespräche und Ausflüge mit dem Egerer Polizeirat Joseph Sebastian Grüner auf der Böhmen-Reise von 1823, vgl. ebd., S. 6of.

15 Johann Wolfgang von Goethe, Elegie von Marienbad. Urschrift. September 1823, hrsg. von Jürgen Behrens und Christoph Michel, mit einem Geleitwort von Arthur Henkel, Frankfurt/Main: Insel 1991. Die Ausgabe bietet dieselben Faksimiles der Kalenderseiten mit Versen und Erläuterungen zum „biographischen Hintergrund“ und zur „Geschichte des Textes".

16 Für die Vermeidung dieser Fokussierung oder die Wahl eines neutraleren Begriffs spricht der alte Verdacht gegen das Abschreiben als „Quelle von Entstellungen, die aus jedem tradierten Text einen verfälschten Text machen“ und entsprechend „Überlieferung“ als „Verfallsgeschichte“ fasst (Davide Giuriato, „Johann Friedrich Oberlin und Herr L... .", in: 


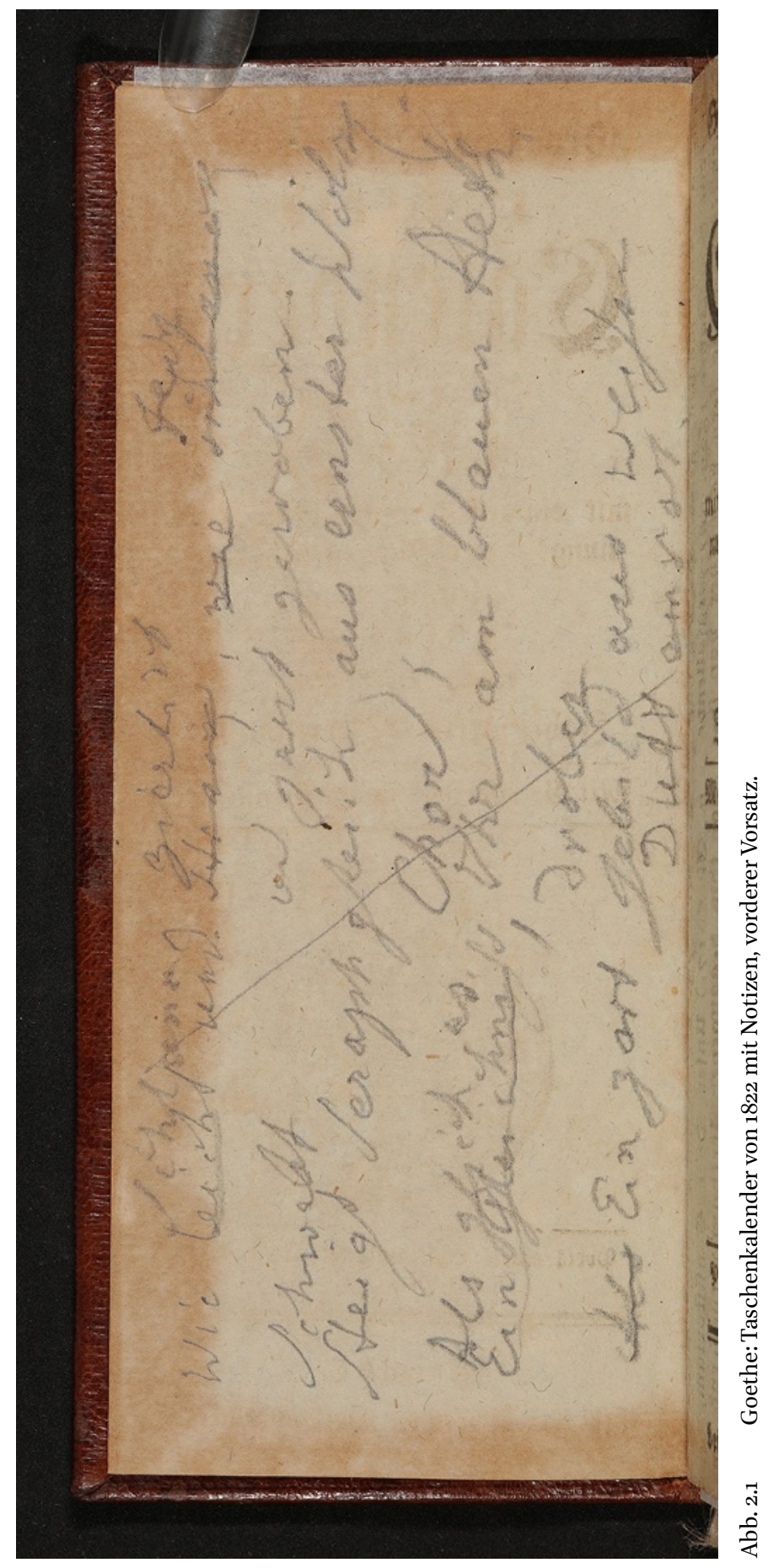


Reduktion des komplexen Schriftgebildes präsentiert, lagert die Varianten in die Anmerkungen aus und gibt, mit Ergänzungen: „Wie schlanck und zierlich, fein und zart gewoben / Schwebt Seraph gleich aus ernster Wolck[en] Chor, / als glich es Ihr am blauen Aeth[er] droben / Ein zart Gebild aus weissem Duft empor.“17 Auf dem Papier steht jedoch: „Wie leicht/schlanck und zierlich/ strack, wie fein/schlanck und zart gewoben / schwebt steigt Seraph gleich aus ernster Wolck[en] Chor, ${ }^{*}$ Als glich es / Ein Gleichnis Ihr am blauen Aether droben * Als ein zart Gebild aus weissem Duft empor" - und tatsächlich kann keine Transkription nachbilden, wie die vielen Umschreibungen des feinen, leichten, zierlichen, zarten, schlanken, duftigen Gebildes sich notdürftig in die Reihe von nur vier Adjektiven zwingen lassen müssen, und wie aus dem noch gleichwertigen schweben und steigen ein eigentümliches emporschweben wird.

Beim Weiterblättern wird man auf insgesamt vierzehn Seiten jeweils ein Verspaar finden, das sich vorerst als Distichon identifizieren lassen würde, bis die Serie mit dem letzten Vers, der eine große Leerstelle nach dem zweiten Wort enthält, vorläufig endet: „Hat liebe an mir geleiste[t]““.18 Danach folgen etliche leere Seiten, nur durchbrochen von einem einsamen waagrechten Bleistiftstrich neben der gedruckten Tabelle für Juli mit beistehenden Namen, auf derselben Höhe wie der Name Christine am 24. Juli. ${ }^{19}$ Gleichsam fünf Monate später, neben der Liste der Tagesdaten für den Oktober, kommt dieselbe Anordnung zu paarweisen Versen in graphisch ähnlichen Schriftzügen dieser vorderen Serie entgegen, nun entsprechend erst lesbar, wenn die Langzeilen um go Grad nach links gedreht werden.

Schlägt man den Kalender auf der Suche nach deren Anfang von hinten auf, findet man aber erstaunlicherweise ein Doppel des Anfangs schon im hinteren Einband, nämlich eine zweite von Stadelmann verfasste, fast nicht mehr lesbare Liste, die mithilfe eines Rechnungsbuches als Verzeichnis von Ausgaben tagesgenau identifiziert werden konnte. Dort sind für den 20. August 1822 unter anderem „Holländisch Pappier" und "Conceptpappier" mit Preisen eingetragen; im Einband fände sich demnach die allerdings zweifelhafte Entsprechung „[Pappier für Concept?]“. ${ }^{20}$ Stimmt diese rekonstruierte Datierung, so wäre der Kalender in seinem vorgesehenen Gebrauchsjahr

Stingelin (Hrsg.), „Mir ekelt vor diesem tintenklecksenden Säkulum“ [Anm. 6], S. 86-101, hier S. 86).

17 Goethe, „Die Urschrift K“ (Anm. 12), S. 19.

18 Ebd., S. $38 \mathrm{f}$.

19 Ebd., [S. 40']. Da die Faksimileedition nur die beschriebenen Seiten aufführt, entspricht die Paginierung nicht der Zählung der Kalenderseiten, sondern dieser Auswahl; die angegebene Seitenzahl in Klammern bezieht sich auf den Kalender selbst.

Ebd., S. $5^{2 f}$. 
1822 für diese Notiz genutzt worden und der Eintrag somit ein Jahr älter als die Gedichtverse vorne. Allerdings werden alle Einträge der Umgebung von den Herausgebern des Kalenders im Verweis auf andere Quellen mit jeweils guter Begründung auf den späten August oder September 1823 datiert. Im selben zeitlichen Raum wie dem der Papier-Anordnung wäre mit dem Hinweis auf die notwendige Anschaffung somit auch ein Vorgang des Schreibens oder Abschreibens skizziert, den die Elegie als besonders aufwendige SchreibArbeit im Spätsommer/Herbst 1823 noch erfahren wird; die Eintragung des zweiten Schreibers bietet somit auch indirekt deren Reflex.

Auf der gegenüberliegenden linken Seite finden sich von Goethes Hand, aber um 18 o Grad gedreht, vierWorte in drei Zeilen, die zweifelsfrei der Reise von 1823 zugeordnet werden konnten: „Sonnabend / Mittwoch / am besten“. ${ }^{21}$ Die nochmalige Wiederholung der Verabredungsnotiz von der ersten vorderen Seite macht jedoch auf eine Eigenart eines anderen Schreibmediums aufmerksam: Während Kalender, ihren expliziten Anweisungen und Anordnungen gemäß, in der Regel dem Jahreslauf folgend gefüllt werden, mit prospektiven Einträgen zu Terminen wie mit retrospektiven Verzeichnungen von Erledigungen und Bemerkenswertem, ist das Notizbuch ein beweglicheres Ding, weil es von vorne und von hinten begonnen werden kann und entsprechend nicht selten Einträge - ohne abgeschrieben worden zu sein - doppelt und dreifach wiederholt vorkommen. ${ }^{22}$ In der fast identischen Wiederkehr der Notiz zu geeigneten Wochentagen bewährt sich geradezu das Prinzip Notizbuch: Als Merkhilfe ist es nicht nur, aber auch ein treuer Speicher, der hier seine Tauglichkeit ausstellt, indem, anders als in den normierten Einträgen zu wechselnden Daten,

21 Ebd. Der Kommentar zu dieser Stelle erläutert schlüssig, dass offenbar eine Verabredung mit Grüner den Gegenstand dieser Notiz bildet, die auch in anderen Schriften entsprechende Spuren hinterlassen hat, vgl. Michel/Behrens, „Erläuterungen“ (Anm. 11), S. $62 \mathrm{f}$.

22 Das Notizbuch zählt somit zur Gruppe der beweglichen Medien, die eigene Schreibformen ermöglichen und generieren, vgl. die Beiträge in Matthias Thiele und Martin Stingelin (Hrsg.), Portable media. Schreibszenen in Bewegung zwischen Peripatetik und Mobiltelefon, München: Fink 2010. Svetlana Efimova fasst entsprechend „vier ausschlaggebende Eigenschaften“ zusammen, die Notizbücher auszeichnen: „Sie sind (1) funktionell gedächtnisstützend und (2) materiell portabel, sie (3) organisieren strukturell einzelne disparate Informationsstücke in eine Sammlung (im Unterschied etwa zu losen Blättern) und sie dürfen / können (4) formell und inhaltlich heterogen sein." Die Hervorhebung des letzten Punkts betont "die volle Freiheit im Umgang mit diesem Medium, die paradoxerweise von seiner gedächtnisstützenden Operativität untrennbar ist“ (Svetlana Efimova, Das Schriftsteller-Notizbuch als Denkmedium in der russischen und deutschen Literatur, Paderborn: Fink 2018, S. 36). Zu ergänzen wäre allerdings, dass das Medium nicht zuletzt wegen seiner materiellen Vorgaben dieser Freiheit immer schon unüberschreitbare Grenzen setzt. 
tatsächlich jeder Einfall und alles schon einmal Erinnerte und wieder Vergessene wie neu verzeichnet ist.

Die lineare Ordnung der Kalendereinträge wird also durchkreuzt von einer anderen Schreib-Logik, die besonders in Prozessen des Skizzierens und Entwerfens zum Einsatz kommt. ${ }^{23}$ Tatsächlich findet man beim nochmaligen Weiterblättern nach einer weiteren kurzen Liste Goethes in der selben Schreibrichtung, , auf dem Kopf stehend“ („Hopfen Saamen / statt Lupulin / tragend“), ${ }^{24}$ wie schon beim ersten Durchgang, hinter diesen Notizen Verse in Langzeilen, die nun für die Lektüre in Gegenrichtung gedreht werden müssen, nämlich um 9o Grad nach rechts, der Transkription zufolge: „Wo Tod und Leben grausam sich bekämpfen / Wohl gäbs ein Kraut des Körpers Quaa[1] zu stillen“25 (Abb. 2.2). Die Schriftzüge sind mit stark aufgedrücktem Bleistift in relativ großen, unsicheren Zügen mit mehreren Spontankorrekturen und kleinen Schreibfehlern im bekannten graphischen Format der abgesetzten Doppelverse auf das Papier gesetzt; erschüttert und erschütternd: „Wo Todeskam[p]f erneuend sich“, gibt die erste Zeile zu lesen, mit Weißraum an ihrem rechten Ende; die Fortsetzung hat offenbar zunächst gelautet: „und Leben sich mit Macht / bekämpfen“. ${ }^{26}$

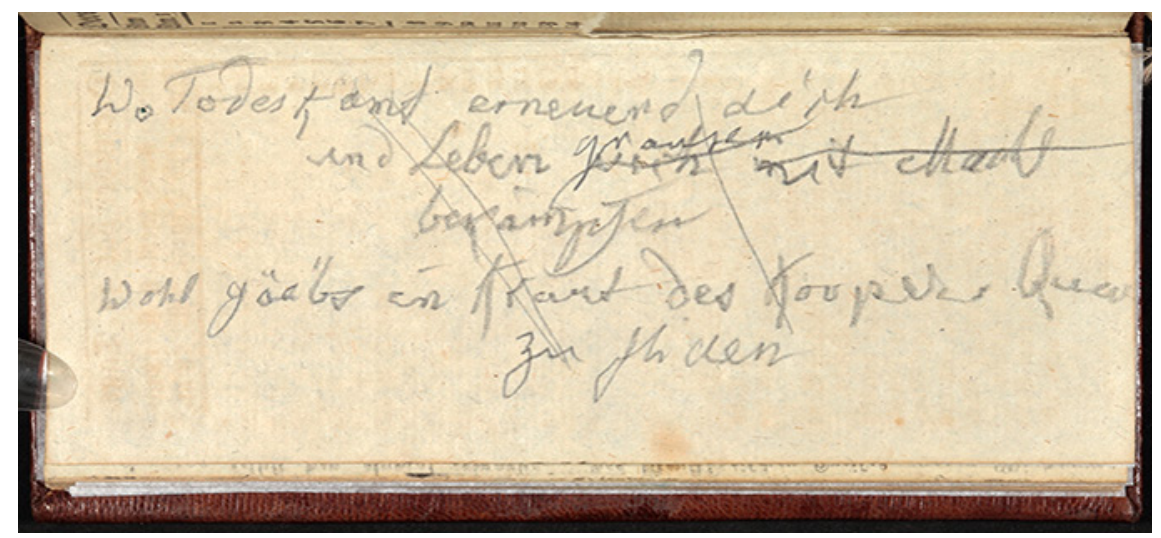

Abb. 2.2 Goethe: Taschenkalender von 1822 mit Notizen.

23 Vgl. Christoph Hoffmann, „Festhalten, Bereitstellen, Verfahren der Aufzeichnung“, in: ders. (Hrsg.), Daten sichern. Schreiben und Zeichnen als Verfahren der Aufzeichnung, Zürich: diaphanes 2008, und die Beiträge in Karin Krauthausen und Omar Nasim (Hrsg.), Notieren, Skizzieren. Schreiben und Zeichnen als Verfahren des Entwurfs, Zürich: diaphanes 2010.

24 Goethe, „Die Urschrift K“ (Anm. 12), S. 5of.

25 Ebd., S. 49.

26 Ebd. 
Wie es bei schnellem Formulieren und Niederschreiben häufiger passiert, hinkt hier augenscheinlich die Ausführung dem Konzept hinterher oder werden sogar zwei Konzepte gleichzeitig geschrieben. ${ }^{27}$ Über diesem vorläufigen Ende des zweiten Anfangs findet sich, nicht nur graphisch auffällig anders, sondern auch Ton und Gestus variierend, das Doppelverspaar: „Was soll ich nun vom Wiedersehen hoffen / Das Paradies, die Hölle steht dir offen. Kein Zweifeln hier! Sie tritt ans Himmels Thor / Und hebt zu ihren Armen dich empor.“28 Diese Verse sind mit guten Gründen als die chronologisch ersten dieses Kalenders und der Elegie identifiziert worden; man sieht sofort, dass zumindest die Schreibsituation, in der sie entstanden sind, offenbar von der ihrer Vorgänger auf der Rückseite komplett verschieden ist. Denn wie die Autopsie der unterschiedlichen Handschriften von derselben Schreib-Hand deutlich macht, sind die meisten Verse im Kalender offenbar tatsächlich unter den erschwerten Bedingungen unterwegs, bei einer oder mehreren Kutschfahrten verfasst worden. Die regelmäßigen stoßweisen Erschütterungen der Schriftzüge und ihre notgedrungen unterschiedliche Größe und Form lassen diese Ausnahmesituation des Schreibens deutlich erkennen. ${ }^{29}$ Außerhalb der Kutsche wäre demnach nur dieses erste Quartett verfasst worden, bzw. lässt sich vielmehr umgekehrt mit Sicherheit sagen, dass es nicht in einem solchen rüttelnden und schwankenden Schreibraum auf einer wackelnden Unterlage verfasst worden sein kann. ${ }^{30}$

Die Urszene des Gedichts wäre somit die des antizipierten Wiedersehens, die im vorderen, nun zweiten bzw. dritten Anfang mit der Beschwörung des emporschwebenden Himmelswesens eine sinnfällige Wiederholung findet. Auch die harte Kontrastierung von Paradies und Hölle, die in den hinteren ersten Versen gleichermaßen beim Wiedersehen drohen, ist in gewisser Weise

27 Vgl. zu einer detaillierten Lektüre der einzelnen Verse und ihrer Varianten Ortlieb: „Notieren, Billettieren, Übereignen“ (Anm. 12).

28 Goethe, „Die Urschrift K“ (Anm. 12), S. 49.

29 Volker Neuhaus hat allerdings darauf hingewiesen, dass ihr eine gewisse Planung vorausging: Goethe habe seine Reisekutsche „eigens mit einem klappbaren Schreibbrett [...] ausstatten lassen, wie man es heute noch in der Remise des Goethehauses betrachten kann" (Volker Neuhaus, Andre verschlafen ihren Rausch, meiner steht auf dem Papiere. Goethes Leben in seiner Lyrik, Köln: DuMont 2007, S. 343.

30 Die Herausgeber des Kalenders bezeichnen sie entsprechend als „dies[e] Initiale des Gedichts, die Goethe von den folgenden Strophen immer deutlich abgesetzt, der er in der Reinschrift einen eigenen Raum gegeben hat", und sie betonen zu Recht, dass dort „im Wiedersehen selbst das Risiko des Umschlags von Paradies in Hölle beschlossen [ist] - und in der frühen Fassung noch rascher, vorbehaltloser zugunsten der Hoffnung entschieden“ (Jürgen Behrens und Christoph Michel, „Nachwort“, in: Goethe, Elegie von Marienbad [Anm. 8], S. 133). 
vorne kryptisch wiederaufgenommen, wenn die zarte schwebende Gestalt mit einem "Seraph“ verglichen wird. ${ }^{31}$ Das „Emporheben“ in dieser hinteren Viererstrophe antwortet somit dem entsprechend weit entfernten Binnenreim des „Emporschwebens"vorne, und wie die Vision Jesajas mit einem schmerzhaften und erlösenden Kuss endet, so wird auch die Reihe der Verse vorne mit einem solchen Kuss vorläufig beendet, der im epischen Präteritum zugleich eine Verheißung der Zukunft ist: „Und mich von da so stufenweis beglückte / Mich nach dem letzten Kuss mich noch ereilte / Den letztesten mir auf die Lippen drückte". 32

Auch hier ist es somit offensichtlich der zum Notizbuch umfunktionierte Kalender, der es erlaubt, die je einzelnen Verse oder Versgruppen gleichzeitig präsent zu halten und das Weiterschreiben nicht linear, sondern im Vor- und Zurückblättern, im Anknüpfen und Abbrechen modellieren zu können. Anders als auf fliegenden Zetteln sind die Verse und Strophen damit aber zugleich auch bereits in eine bestimmte Buch-Ordnung gebracht, die an mehreren Stellen nachweislich auf die Kalendervorgaben auch inhaltlich reagiert. So etwa, wenn ein weiteres isoliertes, abschließendes Verspaar lautet: "Und wundert sich dass nicht um ihretwillen / Die Sonne stille steht", womit die Vorgabe des Taschenkalenders aufgenommen ist, der auf Papier auch den Sonnenumlauf abbildet, weil das Maß des Gregorianischen Kalenders das Sonnenjahr ist, die Zeit, die die Erde braucht, um bei ihrem Weg um die Sonne den Punkt der Frühlings-Tag-und-Nacht-Gleiche zwei Mal zu passieren. ${ }^{33}$

Insgesamt vier Strophen, die allerdings nicht direkt hintereinander folgen und teils größere frei gelassene Leerflächen enthalten, sind auch den Eigenheiten der Landschaft gewidmet, die gleichfalls als Erinnerungsbild in elegischem Ton aufgerufen werden, so dass in diesen Einträgen ebenso die Kalender-Aufforderung zur zeitlich exakten Mitschrift in den getakteten Maßen von Jahr und Monat, Tag und Stunde realisiert ist wie die NotizbuchErmutigung, abseits der Vorgaben bestimmter Textsorten und Gattungen ungeordnet zu verzeichnen, was dann in einem nächsten Arbeitsschritt sortiert

31 Der Prophet Jesaja hat diesen Engeln ihre eigentümliche Gestalt als komplexe Flügelwesen gegeben, wie er sie in einer Vision geschaut hat: „Im Todesjahr des Königs Usija sah ich den Herrn. Er saß auf einem hohen und erhabenen Thron. [...] Serafim standen über ihm. Jeder hatte sechs Flügel: Mit zwei Flügeln bedeckten sie ihr Gesicht, mit zwei bedeckten sie ihre Füße und mit zwei flogen sie. Sie riefen einander zu: Heilig, heilig, heilig ist der Herr der Heere. Von seiner Herrlichkeit ist die ganze Erde erfüllt" (Jesaja 6, 1-7).

32 Goethe, „Die Urschrift K“ (Anm. 12), S. 29.

33 Ebd., S. 44f. 
und umgeschrieben werden wird. Denn schließlich können Aufzeichnungen zu „Fels“, „Busch“ und „Matten“, wie sie der Kalender in (halben) Versen enthält, auch die Gegenstände der Betrachtung fixieren, die der Naturforscher Goethe mit Unterstützung durch Stadelmann und andere besonders aufmerksam betrachtet hat - wie auch die Himmelsphänomene, die in anderer Gestalt Gegenstand der ersten Verse über die ferne Geliebte sind.

\section{II.}

Mit dem zweiten, täuschend ähnlichen und teils identischen Taschenkalender von 1823 , der als noch aktuelles Terminverzeichnis gleichfalls ein nützlicher Begleiter der Böhmen-Reise im Sommer 1823 war, wird auch die Komplexität des Schreibprozesses, als dessen Ende für gewöhnlich die Elegie gilt, sichtbar. Denn wie bereits die wenigen zitierten Verse und Strophen zeigten, ist das Gedicht, das von einer abwesenden Geliebten, der Hoffnung auf ein Wiedersehen, von vergangenem und künftigem Schmerz und von einem Kampf auf Leben und Tod handelt, offensichtlich in mehrfacher Hinsicht datiert und adressiert. Sein autobiographischer Zug liegt erkennbar darin, eine schwere Erkrankung vom Jahresbeginn 1823 einerseits als Zäsur zu markieren, andererseits in die kalendarische Ordnung einzutragen, in die sich auch die alljährlich im gleichen Zeitraum liegende Böhmen-Reise fügt, die ihrerseits in ihren Reisedaten die lebensentscheidende Italien-Reise ab September 1786 zitiert. Dass der Herbst 1823 nach den turbulenten Ereignissen in Marienbad und Karlsbad und eingedenk der lebensbedrohlichen Krankheit des Jahresbeginns auch noch mehr als in früheren Jahren dem 74-jährigen zugleich die Endlichkeit des Lebens und seine eigene Sterblichkeit als andere kalendarische Größen vor Augen geführt hat, scheint nach verschiedenen Zeugnissen evident zu sein. ${ }^{34}$

Entsprechend ist es besonders interessant, dass der Kalender von 1823 auch eine Bilanz enthält, ein Resümee in tabellarischer Form, das gleichermaßen typische Formate von ,Lebenslauf' und ,Publikationsliste' erkennen lässt, eingefügt in unterschiedliche Notizen auf insgesamt fünfzehn beschriebenen Seiten, von denen eine als Eintragung einer anderen Schreibhand identifiziert

34 Die auch in dieser Hinsicht einzigartige Dichte und Fülle historischer Überlieferung zeigt sich etwa in den zusammengestellten Äußerungen von Goethe selbst und von Zeitgenossen, die es erlauben, einzelne Lebensjahre Goethes tagesgenau zu rekonstruieren. Vgl. Johann Wolfgang Goethe, Begegnungen und Gespräche, hrsg. von Ernst Grumach und Renate Grumach, Bd. 14: 1823-24, Berlin, Boston: de Gruyter 2011. 
worden ist. Auffälligerweise mit Tinte geschrieben, steht etwa in der Mitte des Kalenders, zwischen den Seiten mit eigenhändigen Bleistiftnotizen Goethes, in französischer Sprache und zierlicher Schreibschrift in eigenwilliger Schreibweise: „La belle Tragedie Ludgarda, est ecrit par / le General Kropinski auteur de plusieurs / pieces charmantes dela literature polonaise“ („Die schöne Tragödie Ludgarda ist geschrieben von dem General Kropinski Autor mehrerer charmanter Stücke der polnischen Literatur"). ${ }^{35}$ Den Herausgebern des Kalenders von 1822 gebührt das Verdienst, im Anhang ihres Kommentarbands „die noch nie im Zusammenhang edierten Eintragungen in den Kalender für $1823^{\text {“36 }}$ transkribiert und in diplomatischer Umschrift veröffentlicht zu haben. Ihnen zufolge stammt die zitierte Notiz von Graf Constantin von Piotrowski, der als Kurgast in Marienbad war und sie „wohl am 4.8.1823 in den Kalender eingeschrieben" habe, wie ein entsprechender Hinweis in Goethes Tagebuch nahelegt. ${ }^{37}$ Die Formel vom „charmanten Stück“ war ja bereits in den ersten Einträgen des Kalenders von 1822 zu finden gewesen, dort mit der mehrdeutigen Ergänzung „von einem Manne“, die im Kommentar zu dieser Stelle als „verkürzte, andeutende Formulierung“ für eine spätere Erläuterung Goethes bezeichnet wird: Aus seinem „Hang " zu Ulrike von Levetzow könne ein Dramenautor wie Iffland „ein charmantes Stück [...] fertigen, ein alter Onkel, der seine junge Nichte allzuheftig liebt" ${ }^{38}$ Abseits von einer solchen intertextuellen Verflechtung ist die augenfällige Nähe der beiden Formulierungen auch ein Indiz dafür, dass die beiden Kalender tatsächlich parallel oder mit kurzem zeitlichem Abstand als Notizbücher genutzt wurden. Solche quasi unbemerkten Wiederholungen sind wiederum auch in anderen Kontexten unter den typischen Schreibformen des Notizbuchs häufiger anzutreffen. ${ }^{39}$

35 Jürgen Behrens und Christoph Michel, „Anhang. Grosherz. Weimarischer Schreib= Calender für das Jahr 1823 (S. 24)“, in: Goethe, Elegie von Marienbad (Anm. 8), S. 141-152, hier S. 144.

36 Behrens/Michel, „Nachwort“ (Anm. 30), S. 131.

37 Behrens/Michel, „Anhang“ (Anm. 35), S. 15 o.

38 Michel/Behrens, „Erläuterungen“" (Anm. 11), S. 63.

39 Dies gilt ebenso für die berühmten „Schreibbücher“ Jean Pauls, die mit Exzerpten gefüllten selbstgenähten Hefte, in denen Jean Paul, paradox, das ,Abschreiben mit eigenen Worten' zur Anlage großer Materialiensammlungen nutzt, und auch für die „Kladden“ Friedrich Heinrich Jacobis. Beide exzerpieren und kommentieren teilweise dieselben Stellen mehrmals, vgl. zu Jean Pauls entsprechenden Techniken Michael Will, „Jean Pauls (Un-)Ordnung der Dinge“, in: Jahrbuch der Jean Paul Gesellschaft 41 (2006), S. 71-95, und Cornelia Ortlieb, „Jean Pauls Punktiermanier“, in: Jutta Voorhoeve (Hrsg.), Welten schaffen. Zeichnen und Schreiben als Verfahren der Konstruktion, Zürich: diaphanes 2011, S. 77-95. Zu Jacobi vgl. Yves Radrizzani, „Jacobis Auseinandersetzung mit Fichte in den ,Denkbüchern“', in: Fichte-Studien 14 (1998), S. 43-62. 
Einmal mehr lässt das Ensemble von Schriftträgern, Schreibgeräten und Graphemen auch hier erkennen, wie wenig ausschließlich oder zielgerichtet entwerfendes Schreiben vor sich geht. Denn dessen Eröffnung auf den ersten vier Seiten des ,Notizbuch'-Kalenders gilt offensichtlich der Arbeit an Gedichtversen, von denen wiederum die Notizen auf drei Seiten zweifelsfrei demselben Gedicht zugeordnet werden können, weil sie Varianten in Abschriften bilden. Wie in den Auftakt-Versen über das entschwebende Himmelwesen im Kalender von 1822 finden sich auch hier Schriftzüge, die mit Bleistift offensichtlich von Goethes Hand geschrieben sind, in gebrochenen Zeilen und als eine Art erster Strophe, im gleichen Schreibformat auf der um go Grad gedrehten Seite, aber in nur drei gut lesbaren Versen. Sie sind unkorrigiert und sozusagen in Reinschrift, aber vorzeitig abgebrochen: „Ein Dichter glaubt er macht was guts / Doch manchmal zweifelt er am Werthe / Doch seiner Schoenen frischen Muths $[\ldots]^{\text {“. }}{ }^{40}$

Im fehlenden vierten Vers ist hier performativ realisiert, was der zweite Vers mit dem Zweifel am Wert schon artikuliert hatte, entsprechend also auch der erste Vers mit seiner vorsichtigen Zuversicht performativ dementiert: die Selbstermutigung des Dichters und der obsiegende Zweifel an der Qualität des Gemachten, mit der Konsequenz seines sofortigen Abbruchs. Grund dafür ist womöglich eine Art Kopierfehler: Die anaphorische Wiederholung des Gegenworts „Doch“ gibt zwar eine rhetorische Bekräftigung, mag aber auf so engem Vers-Raum für zu unpoetisch befunden worden sein; über andere Gründe für die Löschung des virtuellen vierten Verses vor seiner Niederschrift lässt sich nur spekulieren..$^{41}$ Beim stereoskopischen Blick auf beide Kalender, die im Raum- und Zeitgefüge der historischen Reise zusammengehören, ist in der "Schoenen“ des dritten Verses und der Formel vom „frischen Muth“ auch eine Doppelung des adressierten zarten Himmelswesens zu sehen - beide Versgruppen zitieren und kommentieren einander unmittelbar, wenn sie, wie einstmals, auf einem Schreibtisch oder dessen improvisiertem Ersatz nebeneinander liegen.

Beim Weiterblättern gibt auch der zweite Taschenkalender dann weitere Verse zu lesen, in denen ein entscheidendes Wort dieser ersten Seite im Abschreiben variiert ist, allerdings wiederum in einem Entwurf, den man auf

40 [Johann Wolfgang Goethe,] „Ein Dichter glaubt er macht was guts“, in: Goethe, Gedruckter Weimarischer Kalender (Anm. 8), unpag., [S. 2 / Titel].

41 Die paradoxe Formulierung von der, Löschung vor der Niederschrift‘ trägt dem Umstand Rechnung, dass es vielfältige Varianten des Streichens und Tilgens mit je eigenen Implikationen und Funktionen gibt, vgl. die Beiträge in Lucas Marco Gisi, Hubert Thüring und Irmgard M. Wirtz (Hrsg.), Schreiben und Streichen. Zu einem Moment produktiver Negativität, Göttingen: Wallstein 2011. 
den ersten Blick als mehrfach überschrieben erkennt (Abb. 2.3): „Sonst schien ich lieb und werth zu seyn" - das übernommene Wort aus den ersten drei Versen ist also „werth“, nun vom Geschriebenen auf den Schreiber gewendet. ${ }^{42}$ Entsprechend pointiert gestaltet sich der Anfang in der überschriebenen Fassung: „Ich schien lieb und werth zu seyn“, allerdings mit dem Effekt, dass das zweite gestrichene Wort des ersten Verses gleichfalls „ich“ lautet und eine Lücke entsteht, in die nach Versmaß und Sinn eigentlich ein "dir" gehören würde. ${ }^{43}$ Auch diese Anrede im zweiten Vers ist zweifach modelliert: „Du liebtest noch“ ist gestrichen, neutraler, sachlicher heißt es nun: „Und dir gefiel die kleinste Gabe“. ${ }^{44}$ Beide Streichungen nehmen zudem den Versen ihren Zeit-Index: Nicht „sonst“ schien „ich“ und nicht "noch“ liebte „du“ - allerdings ist dieses „noch“ quasi in den dritten Vers kopiert, mitsamt dem Zweifel, den der Vers explizit macht: „Und wenn ich deine Gunst noch habe / So sind die Tafeln nicht zu klein. ${ }^{45}$

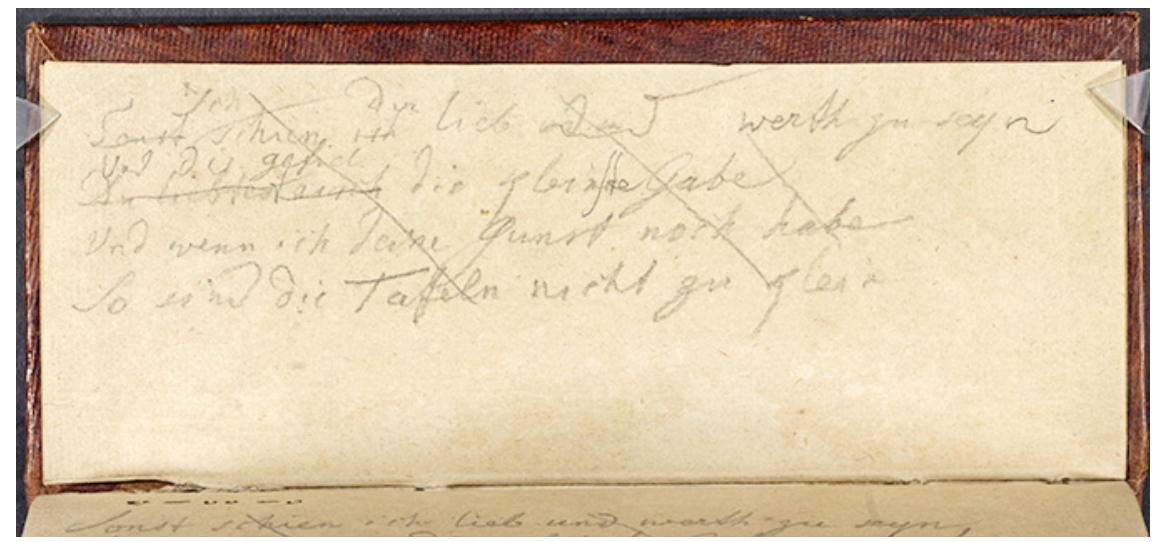

Abb. 2.3 Goethe: Taschenkalender 1823 mit Notizen.

Auch hier sind die schrägen Striche von links oben nach rechts unten, die das gesamte Gebilde doppelt durchstreichen, von besonderem Interesse, denn sie zeigen wie üblich eine Erledigung an, hier die Abschrift auf der nächsten Seite in vier Versen, die zugleich umgeschrieben sind: „Sonst schien ich lieb und werth zu seyn“, nun wieder mit „ich“ an seinem alten Platz, womöglich, um das

42 Goethe, Gedruckter Weimarischer Kalender (Anm. 8), S. 4.

43 Ebd.

44 Ebd.

45 Ebd. 
sonst nötige "du“ im Vers zu vermeiden. ${ }^{46}$ Gleichermaßen bereits im Abschreiben geändert ist der zweite Vers, der allerdings sofort wieder überschrieben wird: „Auch dir gefiel die kleinste Gabe“ wird zu: „Dich freute selbst die kleinste Gabe“. ${ }^{47}$ Identisch übernommen ist dann der dritte Vers, aber wieder wird überschrieben: „Und wenn ich deine Gunst noch habe“ ist an einer Stelle versmaß-neutral, aber semantisch entscheidend verändert im „noch“ $\mathrm{zu}$ „nur", so dass sich ergibt: „Und wenn ich deine Gunst nur habe“. ${ }^{48}$ Erst der nachgeschobene Vers macht einen Bedingungssatz aus dem Verspaar: Wenn die Gunst gehabt wird, „sind die Tafeln nicht zu klein“, vielmehr, noch stärker in der Sofort-Korrektur im Überschreiben und Ergänzen zur hyperbolischen Formel: „So ist kein Täfelchen zu klein“.49 Dieser im Kontext etwas paradoxe Superlativ in der Litotes des, nicht zu Kleinsten' nimmt auf, was der zweite Vers gleichfalls im Superlativ formuliert hatte: Ihr, der Adressatin, „gefiel die kleinste Gabe". Somit wird in der galanten Überbietung bisherigen Gebens auch die allerkleinste akzeptiert werden - und entsprechend bescheiden nimmt sich die Hoffnung auf die gewährte Gunst aus (Abb. 2.4).

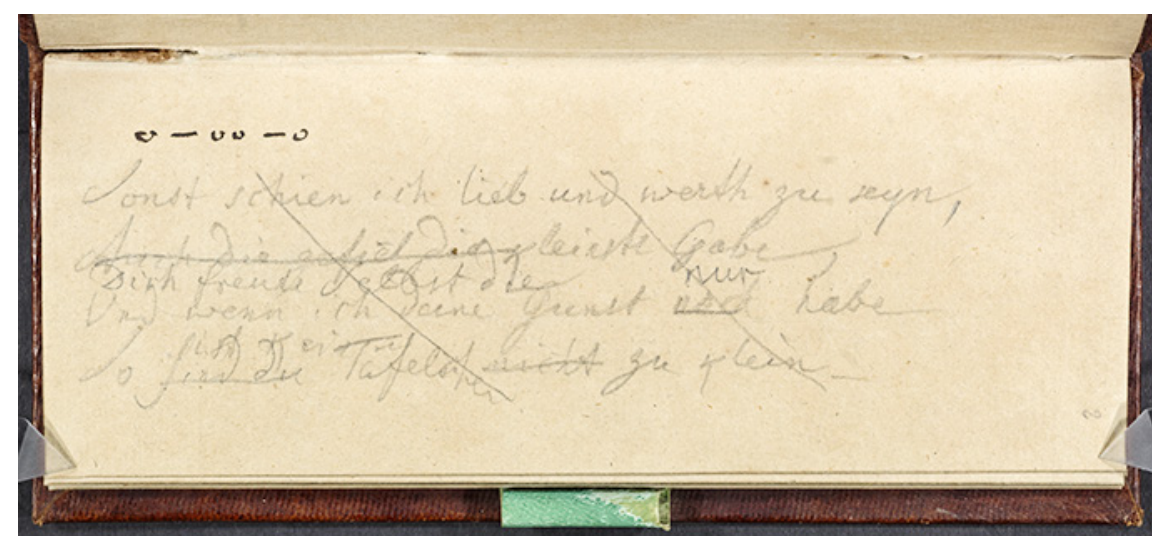

Abb. 2.4 Goethe: Taschenkalender von 1823 mit Notizen.

Gestrichen und ersetzt wurden aber auch diese Verse, so dass man noch einmal weiterblättern muss und nun vier unkorrigierte, ungestrichene Verse vor Augen hat, erstmals auch mit Satzzeichen und einer gleichförmigen GroßSchreibung der Anfangsbuchstaben, aber auch hier wurde im Abschreiben

\begin{tabular}{ll}
\hline 46 & Ebd., S. 6. \\
47 & Ebd. \\
48 & Ebd. \\
49 & Ebd.
\end{tabular}


quasi unmerklich geändert, und man ist nun genügend sensibilisiert für die kleinsten Verschiebungen in diesem kopierten Gefüge: „Gewogen schienst du mir zu seyn, / Du lächeltest der kleinsten Gabe. / Und wenn ich deine Gunst blos habe / So ist kein Täfelchen zu klein“.50 Diese Variante bittet quasi um Nachsicht für die Kleinheit der Gabe, Gunst und Gabe sind wieder enger aufeinander bezogen, galant, aber vollkommen unanstößig, zumal das neu eingesetzte "blos“ auch die Feststellung der gehabten Gunst wieder höflich zurücknimmt. Ebenso wird nach der vormaligen Freude und Liebe der Beschenkten nun nur noch gewissermaßen gesellschaftlich neutral ihr vergangenes Lächeln vermerkt, wie auch das sprechende Ich erst im dritten Vers eingeführt wird, anstatt wie in den vorigen Fassungen im ersten Vers oder gar als dessen erstes Wort aufzutrumpfen. Diese insgesamt zurückgenommenen Verse haben offenbar tatsächlich eine Gabe begleitet oder angezeigt, gemeint sind wohl kleine Schokoladetafeln, wie sie für Ulrike von Levetzow und ihre beiden Schwestern im vorigen Sommer schon zwischen solchen aus Stein verborgen lagen und offenbar als Belohnung für ihr Interesse an gesteinskundlichen Erläuterungen in Marienbad gedacht gewesen waren. ${ }^{51}$ Auch hier demonstriert die Spur des Schreibprozesses wieder die enge Zusammenarbeit und wechselseitige Beeinflussung eines Kollektivs von Schreibgrund, Werkzeug und Versformat: Spontan korrigiert im Medium des Entwurfs, der Bleistiftskizze, sind die solchermaßen beweglichen Anordnungen der einzelnen Worte doch stillgestellt durch ihre stabile Anordnung zu Viererblöcken auf je einer neuen Seite, die jeweils noch genau so viel Leerraum enthält, wie ihn die offenbar wenigen erwarteten Korrekturen benötigen würden. Ihren fixierten Ort haben die Schriftzüge des Kalendergedichts zur Schokoladengabe schließlich auf einem jener Billetts für Ulrike von Levetzow gefunden, die auch mit

$50 \quad$ Ebd.

51 Die Begleitverse zur Schokoladengabe von 1822 wurden mit entsprechender Adressierung und Datierung in die Propyläenausgabe aufgenommen: „An Ulrike von Levetzow / Marienbad, Juli 1822 / Genieße dies auf deine eigene Weise, / Wo nicht als Trank, doch als geliebte Speise“ (Johann Wolfgang Goethe, „An Ulrike von Levetzow“, in: ders., Sämtliche Werke. Propyläenausgabe, hrsg. von Curt Noch, Berlin: Propyläen [1909-32], Bd. 35, S. 211. Auch die Widmungsverse zur Überreichung seines Geschenks von Dichtung und Wahrheit, mit Bezug auf den Bericht des Champagne-Feldzugs finden sich dort, unmittelbar im Anschluss: „An Ulrike von Levetzow / Wie schlimm es einem Freund ergangen, / Davon gibt dieses Buch Bericht. / Nun ist sein tröstendes Verlangen: / Zur guten Zeit vergiß ihn nicht!“ (Ebd., S. 212) Beide Gaben und Versgruppen integriert auch Friedemann Bedürftig in seine fiktionale Ausmalung der historischen Szene (Friedemann Bedürftig, Die lieblichste der lieblichsten Gestalten. Ulrike von Levetzow und Goethe, Reinbek bei Hamburg: Rowohlt 2004, S. 12). 
entsprechend explizitem Titel in der Propyläenausgabe Goethes in Auswahl veröffentlicht wurden. ${ }^{52}$

Demgemäß lassen sich auch andere Einträge im Kalender, die offensichtlich Verscharakter haben, als solche Entwürfe von Gedichten für Ulrike von Levetzow lesen, etwa die unmittelbar auf der Rückseite der letzten Fassung des „Täfelchen“-Gedichts anschließenden, ausnahmsweise mit Tinte und somit dauerhaft geschriebenen Verse von Goethes Hand: „Ich bin gefangen! Wie! In tiefer Gruft, / So wär ich alsobald in freyer Luft". ${ }^{53}$ Erich Trunz hat sie "fragmentarisch[e] Zeilen“ genannt und auf ihren besonderen Ort im "Schreibkalender des Jahres 1823“ hingewiesen: „Goethe hat diese Verse versteckt, aber nicht vernichtet. “54 Auf einer der letzten beschriebenen Seiten findet sich zudem ein weiteres, zweifellos an Ulrike von Levetzow adressiertes Gedicht, das in den gedruckten Werk-Ausgaben Goethes in der Regel den dritten Text der Rubrik An Ulrike von Levetzow ausmacht: „Dann senkt sich grau / und imer gra[uer] / aus Wolke[n] Schich[t] / ein Regen Schauer // Weisst du ob's / heitert / ob es reg[net] // Du Schüler Hobarts Wund[er]lich“. ${ }^{55}$ Während die veröffentlichte Fassung Du Schüler Howards, wunderlich mit der Adressierung des letzten Verses beginnt und den suchenden Blick in die Umgebung und zum Himmel, „ob Nebel fallen, ob sie steigen, / Und was sich für Gewölke zeigen“, zum Ausgangspunkt für einen Weitblick in die Bergwelt und die Rückwendung $\mathrm{zu}$ „holder Schwelle“ und einem „allerliebste[n] Treugesicht“ nimmt, ${ }^{56}$ setzen die Verse dieses Entwurfs das Wetter- und Stimmungsbild an den Anfang und ergänzen die entsprechende doppelte Frage, die sich ebenso auf den Sprecher zurückwenden lässt, der sich hier und andernorts im „Du“ auch selbst anredet.

$5^{2}$ Unter der Sammelüberschrift „An Ulrike von Levetzow“ mit einer pronominalen Wiederholung im Untertitel „An dieselbe“ steht dort „Gewogen schienst du mir zu sein, / Du lächeltest der kleinsten Gabe; / Und wenn ich deine Gunst nur habe, / So ist kein Täfelchen zu klein“ (Goethe, „An Ulrike von Levetzow“ [Anm. 51], S. 9).

53 Behrens/Michel, „Anhang“ (Anm. 35), S. 144.

54 Erich Trunz, „Goethes späte Lyrik“, in: ders., Ein Tag aus Goethes Leben. Sonderausgabe, München: Beck 2006, S. 147-166, hier S. 15o. Auch im Kommentar der von ihm herausgegebenen Werkausgabe spricht Trunz vom „Kreis der Elegie“ und von der „Ulriken-Lyrik“, plädiert also dafür, die einzelnen Gedichte nicht für sich, sondern in diesem doppelten Kontext zu lesen. Dazu gehören für Trunz auch die drei stets vor der Gruppe „An Ulrike von Levetzow“ stehenden „Kurzgedichte“ „Könnt ich vor mir selber fliehn!“, „Ach! Wer doch wieder gesundete!“ und „Denn freilich sind's dergleichen Kiel' und Pfeile“ (Goethes Werke [Anm. 10], Bd. 1: Gedichte und Epen, S. 754).

55 Behrens/Michel, „Anhang“ (Anm. 35), S. 146.

$5^{6}$ Goethes Werke (Anm. 10), Bd. 1, S. 378 . 


\section{III.}

Buchstäblich zwischen diesen Versuchen der adressierten poetischen Rede an ein fernes Gegenüber, in dem man mit einiger Sicherheit Ulrike von Levetzow sehen kann, steht ein anderes Medium der (Selbst-)Vergewisserung, die bereits erwähnte Tabelle (Abb. 2.5). Einmal mehr erfüllt sich hier auch die Vorgabe des formatierten Schriftträgers, denn die gerahmte Auflistung in Zahlen und Stichworten stellt einen eigenen kleinen Kalender im Kalender dar: Jeweils links ist mit arabischen Zahlen von oben nach unten der jeweilige Listenposten nummeriert, an den sich, mit einem Schrägstrich von links unten nach rechts oben zugleich getrennt und verbunden, die jeweils vierstellig geschriebene Jahreszahl unmittelbar anschließt. Das hier angelegte Spaltenformat halten auch die Einträge, bei allen anderen Unterschieden, konsequent ein, indem das erste Notat als Zeileneintrag immer direkt rechts von der Jahreszahl eingefügt ist, während gelegentliche Zusätze in einer zweiten Zeile nach Art einer Einrückung mit etwas freiem Raum links notiert sind. Auch wenn schon die zweite und vierte Eintragung mit Streichungen, offenbar spontanen Ergänzungen und einem offensichtlich späteren Nachtrag graphisch die ruhige Ordnung der Tabelle durchbrechen, zeigen vor allem einmal mehr die Leerstellen, wie das Schreibmaterial solche Formate zugleich ermöglicht und begrenzt. Denn auf die vierte Jahreszahl, „4 / 1797“, folgen senkrecht untereinander, mit einigem Abstand, „1. Briefe / 2. / 3 / 4 Reiseacten“, und entsprechend wäre für die mittleren beiden Zahlen Raum für jeweils zwei oder mehr ganze Zeilen, die andere Schreibarbeiten des Jahres in einem zweiten oder mehrfachen Durchgang hätten aufnehmen können. ${ }^{57}$

Dabei ist überhaupt erst in dieser Tabellenzeile mehr Platz für solche Ergänzungen gelassen, mitsamt der Einführung der zweiten Nummerierung, augenscheinlich nachdem die Text-Ergänzung des Datums „Herm und / Dorothea gedruckt“ zweifach ergänzt und überschrieben wurde, einmal mit dem Wort „Liebe“, im selben Schriftduktus und mit derselben Bleistiftfarbe parallel zu „Herm“ über der Zeile eingetragen, zum zweiten Mal mit dem offenbar zu einem anderen Zeitpunkt mit spitzerem Bleistift in dunklerem Strich später hinzugefügten, unsicher gemalt oder während einer Kutschfahrt erschütterten Wort „Rosla“. ${ }^{58}$ Diese beiden Zusätze legen es nahe, dass die Auflistung von

Goethe, Gedruckter Weimarischer Kalender (Anm. 8), S. 40. Vor der Angabe der Signatur verzeichnet der hier zitierte Katalog des Goethe- und Schiller-Archivs „Schrift: egh [i. e. eigenhändig]“, womit der Status des späten Autographs benannt ist, nicht aber in den Blick geraten kann, dass auch dieser Kalender von mehr als einer Schreibhand beschriftet wurde.

$5^{8}$ Goethe, Gedruckter Weimarischer Kalender (Anm. 8), S. 40. 
Abb. 2.5

Goethe: Taschenkalender von 1823 mit Notizen, Tabelle, 1. Teil.

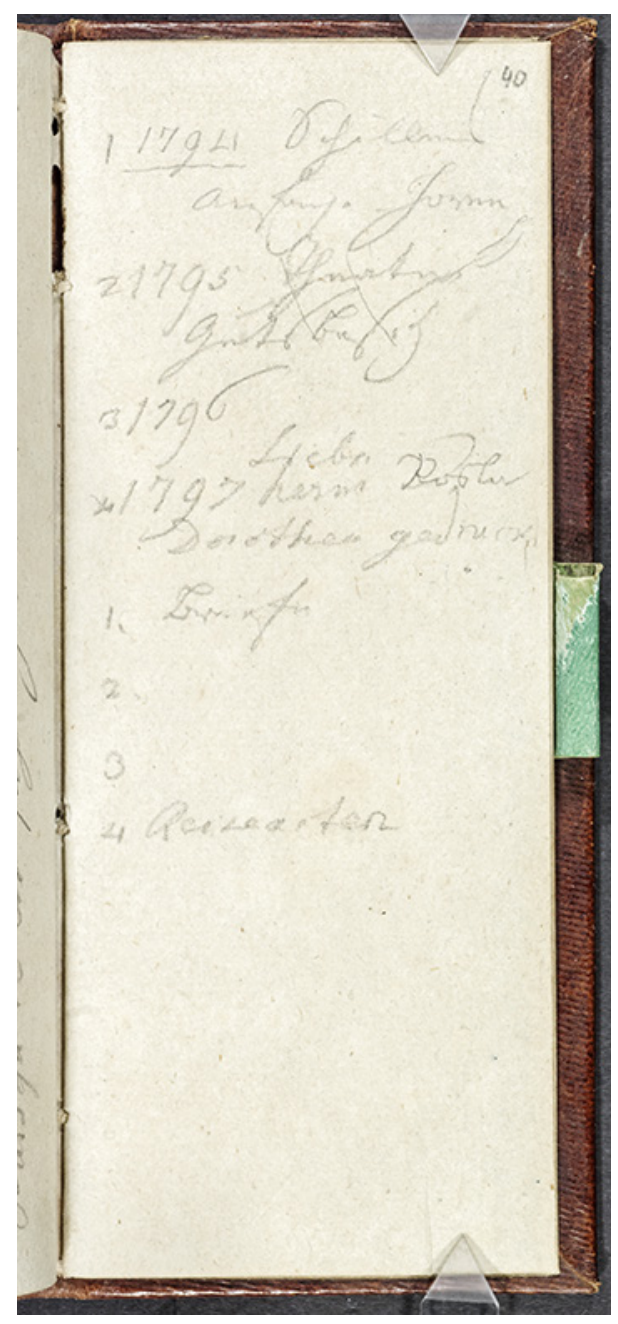

Projekten und Werken um wichtige biographische Stationen ergänzt wurde oder weiterhin ergänzt werden sollte: Das denkbar enigmatische „Liebe“ kann darauf verweisen, dass Goethe seine dritte Schweizer Reise für einen Aufenthalt in Frankfurt nutzte, um Christiane Vulpius, mit der er seit 1788 liiert war, und den gemeinsamen Sohn August, geboren 1789, seiner Mutter vorzustellen; zudem hatte er vor der Reise seine Familie testamentarisch abgesichert.

Und die Kurzform „Rosla“ kann für eine weitere Episode des gemeinsamen Lebens stehen: Goethe hatte in Oberroßla, einem kleinen Dorf zwischen Apolda und Oßmannstedt, von 1798 bis 1803 ein Freigut besessen, das schon 1796 in den Weimarischen Wöchentlichen Anzeigen zum Verkauf ausgeschrieben 
worden war, so dass die Jahreszahl für den komplexen Vorgang der Versteigerung stehen kann, bei dem sich mehrere Bieter nicht einig wurden und der Herzog schließlich persönlich eingriff. ${ }^{59}$ Die erste Erwähnung des Guts im Briefwechsel mit Christiane Vulpius legt es außerdem nahe, das zweite Stichwort auch als Kommentar des ersten zu lesen, denn Goethes kurzer Brief kündigt zunächst seinen heimlichen Besuch bei ihr in Weimar an und schlägt dann einen gemeinsamen Sonntag in Roßla vor, bei dem sie „alles Vorgefallne besprechen [können] " würden. ${ }^{60}$

Eigentlich hatte jedoch bereits die erste Eintragung am oberen Rand der Seite diese untrennbare Verbindung von Schreibprojekten, Werken und biographischen Daten vorgegeben: „1 1794 Schillers / Anfang. Horen“. ${ }^{61}$ Ein passendes historisches Ereignis lässt sich leicht finden: Im Dezember 1794 veröffentlichte Schiller die Ankündigung seines Zeitschriftenprojekts Die Horen, das wiederum den Anlass für den ab Juni 1794 mit Goethe aufgenommenen Briefwechsel bot. Das gemeinsame Schreiben ist dort Programm, denn für das Gelingen der monatlich erscheinenden Schrift soll es laut Schillers Vorwort notwendig sein, "mehrere der verdienstvollesten [sic] Schriftsteller Deutschlands zu einem fortlaufenden Werke zu verbinden, an welchem es der Nation trotz aller Versuche, die von Einzelnen bisher angestellt wurden, noch immer gemangelt hat und notwendig mangeln mußte, weil gerade eine solche Anzahl und eine solche Auswahl von Teilnehmern nötig sein möchte, um bei einem Werk, das in festgesetzten Zeiten zu erscheinen bestimmt ist, Vortrefflichkeit im einzelnen mit Abwechslung im ganzen zu verbinden“. ${ }^{62}$ An zehnter

59 Vgl. zu den Details Adolph Doebber, „Goethe und sein Gut Ober-Roßla. Nach den Akten im Goethe- und Schiller-Archiv und im Geh. Haupt- und Staats-Archiv zu Weimar“, in: Jahrbuch der Goethe-Gesellschaft 6 (1919), S. 195-204.

6o Goethe an Christiane Vulpius, Oberroßla, 21.06.1798, in: Goethes Ehe in Briefen, hrsg. von Hans Gerhard Gräf, Frankfurt/Main: Insel 1921, S. 153. Eine Anmerkung ergänzt, Goethe habe es allerdings vorgezogen, im Gästezimmer des Pfarrers zu wohnen statt auf dem Gut (vgl. ebd.). Mindestens im Jahr 1801 lebten beide zusammen dort, etwa im April 1801 während Goethes längeren Aufenthalts in Oberroßla vom 25. März bis 3o. April.

61 Goethe, Gedruckter Weimarischer Kalender (Anm. 8), S. 4or.

62 Friedrich Schiller, „Ankündigung. Die Horen, eine Monatsschrift, von einer Gesellschaft verfaßt und herausgegeben von Schiller“, in: Schillers Werke. Nationalausgabe, hrsg. von Julius Petersen et al., Bd. 22, Weimar: Böhlau 1943ff., S. 106f., hier S. 107. Vgl. zur Horen-Zusammenarbeit als Beginn der Kooperation von Schiller und Goethe Hartmut Reinhardt, „Ästhetische Geselligkeit. Goethes literarischer Dialog mit Schiller in den ,Unterhaltungen deutscher Ausgewanderten“', in: Peter-André Alt et al. (Hrsg.), Prägnanter Moment. Studien zur deutschen Literatur der Aufklärung und Klassik. Festschrift für Hans-Jürgen Schings, Würzburg: Königshausen \& Neumann 2002, S. 311-341, 
Stelle, nach, unter anderem, „Professor Fichte in Jena“, „Professor Garve in Breslau“ und „Kanonikus Gleim in Halberstadt“ und vor seinem Jugendfreund "Geheimer Rat Jacobi in Düsseldorf" und dem „Hofrat Schiller in Jena“ selbst, findet sich dort auch "Geheimer Rat von Goethe in Weimar" ${ }^{43}$ Steht so gleichermaßen der Freund, dessen „Anfang“ und das kollektive Schreibprojekt der Horen am Anfang der bilanzierenden Chronik, so endet sie am Fuß der nächsten Seite bezeichnenderweise mit einem doppelten Datum und einer leeren Zeile: „1812 Schlacht v. L[eipzig] / 1813“, 64 bevor auf der nächsten Seite das bereits zitierte Gedicht „Dann senkt sich grau“ folgt. ${ }^{65}$

Mehr noch, die Rückseite führt mit einem neuen Format auch neue Inhalte ein (Abb. 2.6): Die Nummerierung in arabischen Zahlen fehlt komplett, dafür sind hier von den insgesamt fünfzehn Jahreszahlen einmal drei und zweimal vier mit den typischen geschweiften Klammern, die sonst das Ende von Versen oder Zeilen graphisch markieren und semantisch bekräftigen, zusammengefasst, wobei nur die letzten vier, die Jahre von 1810-1813, unmarkiert bleiben. Die Reihung knapper Stichworte ist im dritten Vier-Zeilen-Block teils zu wenigen Buchstaben verkürzt; mit besonders auffälligen Leerstellen: „1798. Propyläen / 1799 Ausstellungen / 1800 Sieben. / Acten der d. Theater / 1802 Farbenl. / 1803 / 1804 / 1805 / Schiller stirbt 1806 Octbr. Franz[osen] / 1807 Leide[n]schaft / 1808 Wahlverwdsch. / 1809 Erf[urter] Congress / 1810 F[arben] L[ehre] gedruckt / 1811 / 1812 Schlacht v. L[eipzig] / 1813“.66 Die neue historisch-kritische Ausgabe der Tagebücher wird womöglich diese teils kryptischen Angaben mit einem Kommentar erhellen können; in ihrer bislang edierten Form sind sie lediglich als „das im Tagebuch vom 11. bis 15.8.1823 verzeichnete Sichten und Diktieren der Chronik von 1798 [sic] bis 179 o vorbereitend“ klassifiziert. ${ }^{67}$

zur Gründung der Horen unter Zensur-Bedingungen ders., „,... man weiß nicht, was man schreiben darf ...' Die Weimarer Klassik und die Zensur: Zwei Fallstudien zu Schiller und Herder“, in: Wilhelm Haefs und York-Gothart Mix (Hrsg.), Zensur im Jahrhundert der Aufklärung. Geschichte, Theorie, Praxis, Göttingen: Wallstein 2007, S. 203-224.

63 Schiller, „Ankündigung“ (Anm. 62), S. 107. Vgl. zum „Musenkreis“ als entsprechend sinnfällig gewählter Gemeinschaft der antiken Göttinnen und den Implikationen für das Programm der Zeitschrift Martin Dönike, Pathos, Ausdruck und Bewegung. Zur Ästhetik des Weimarer Klassizismus 1796-1806, Berlin: de Gruyter 2005, S. $12 \mathrm{ff.}$

64 Goethe, Gedruckter Weimarischer Kalender (Anm. 8), S. 40v.

65 Ebd., S. 41r.

66 Ebd., S. 4ov.

67 Behrens/Michel, „Anhang“ (Anm. 35), S. 15o. 


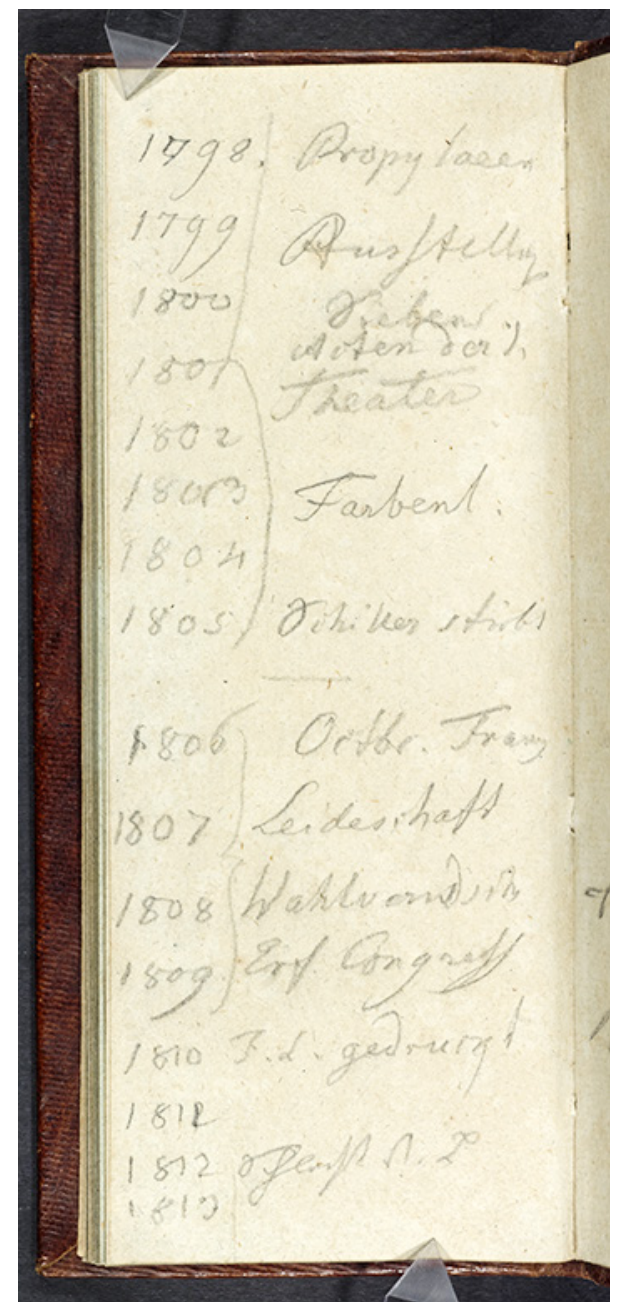

Abb. 2.6

Goethe: Taschenkalender von 1823 , Tabelle, 2. Teil.

Die Tabellendaten setzen aber, wie zitiert, erst mit 1794 ein und enden mit der Leerzeile nach 1813, die sich mit besonderen Begegnungen, etwa mit Zar Alexander I. oder mit Fürst Metternich wiederum im politischen Kontext füllen ließe. 1813 ist aber auch das Jahr, in dem Goethe zum 26. August das allegorische Liebesgedicht Gefunden für Christiane Vulpius verfasst, zur Feier ihrer 25 Jahre zurückliegenden ersten Begegnung, wobei zum mutmaßlichen Zeitpunkt der Eintragung dieser Jahreszahl im August 1823 wiederum zehn Jahre vergangen sind. Zugleich rückt die Datenleiste der Jahreszahl ihres Todes - 1816 - bedenklich näher; mit 1814/15 hätte auch Marianne von Willemer als nicht-genannte Geliebte mit der Hafis-Lektüre, dem Beginn der Arbeit am 
Westöstlichen Diwan, Teil des Verzeichnisses werden können. Dass die Tabelle chiffriert auch solche veränderten emotionalen Verhältnisse verzeichnet, zeigt sich schon beim lakonischen Eintrag „Leide[n]schaft“ für 1807, der womöglich auf die unglückliche Liebe zu Wilhelmine Herzlieb in diesem Jahr verweist. Evident ist eine solche Verschlüsselung schon zuvor im ungewöhnlich genau datierten Eintrag „18o6 Ocbr. Franz[osen]“, der offensichtlich auf die Besetzung Weimars durch napoleonische Truppen und die Gefahr für das eigene Haus rekurriert, aber zugleich auch das Datum der Trauung mit Christiane Vulpius infolge des erschütternden Ereignisses, den 19. Oktober 1806, indirekt anzeigt. Auf dem Erfurter Kongress ist Goethe im Jahr 1808, nicht 1809, wie die Tabelle angibt, Napoleon vorgestellt worden, ein epochales Ereignis, das in diesem Block der Eintragungen möglicherweise gleichfalls wegen seiner besonderen emotionalen Qualität zu stehen kommt - vertauscht mit den Wahlverwandtschaften von 1809, die mit den Oktober-Ereignissen so den Rahmen um das erratische Stichwort „Leide[n]schaft“ bilden.

Hat der erste Block den Charakter einer nüchternen Auflistung von Projekten und Publikationen, so enthält der zweite einen eigenen Spannungsbogen vom hochgestimmten ersten Eintrag „1802 Farbenl.“, der die Arbeit an der Farbenlehre, dem Zentrum von Goethes Forschungen zu Optik und Licht, markiert, zum Abgrund von „1805 Schiller stirbt“, einer Zäsur, die graphisch durch einen durchgezogenen Querstrich verstärkt ist. Erst mit einigem Abstand folgt dann das zitierte 1806-Erlebnis, und entsprechend bildet die sorgfältig in etwas kleineren Buchstaben geschriebene Eintragung nicht zum Tod, sondern zum Sterben Schillers, das Zentrum der Seite, auf das wegen der umgebenden Leere, des Unterstrichs und der auffälligen nach rechts gerichteten Klammern der Blick zuerst fällt. Spätestens diese Stelle erinnert an die einstige, mehrere Tage umspannende Tagebucheintragung Goethes „Leiden und Träumen“ nach dem kurzen Eintrag vom 16. Juni 1777: „Brief des Tods meiner Schwester. Dunkler zerrissener Tag“, gleichfalls umgeben von zwei großen weißen Blöcken Leerraum, zwischen den Leerzeilen nach "17.“ und „19.“68 Goethe und Schiller waren beide seit dem Jahresanfang 1805 schwer krank gewesen, so dass der Vermerk zum Sterben Schillers im Präsens auch an den damaligen eigenen Kampf um Genesung erinnern kann. Zudem ist er aber im Jahr 1823, dem Jahr, das wiederum mit einer schweren Erkrankung Goethes begonnen hatte und, wie der Schreib-Kalender von 1822 festhält, buchstäblich und metaphorisch Herz und Schmerz einmal mehr untrennbar verbindet, auch ein Menetekel

68 Tagebuch vom 1.-22. Juni 1777, in: Johann Wolfgang Goethe, Tagebücher. Historischkritische Ausgabe in acht Bänden, hrsg. von Jochen Golz et al., Bd. I,1, Stuttgart: Metzler 1998ff., S. 42. 
der eigenen Sterblichkeit. Dagegen hat die vollendete Elegie nach mehreren weiteren Abschriften allgemeineren Charakter, zumal sie fast immer (nur) als Teil der Trilogie der Leidenschaft, in die Goethe sie 1825 eingefügt hat, und nicht als Teil des kalendarischen Kollektivs gelesen wird. ${ }^{69}$

IV.

Wie Goethe vorgedruckte kleine Kalender für sein Tagebuch in Prosa, in Stichworten und Sätzen genutzt hat, so adaptiert er auf den leeren weißen Seiten des zum Notizbuch umfunktionierten Taschenkalenders von 1823 das dort übliche Tabellenformat mit entsprechenden Folgen für Art und Format der Einträge. Hatten die Langverse im quer gelegten Kalender von 1822 noch mit den offensichtlichen Beschränkungen für das freie Dichten durch die hochformatigen schmalen Seiten zugleich deren Überwindung dokumentiert, so zeigt umgekehrt der Kalender von 1823, wie die Möglichkeit der freien Gestaltung leerer Seiten zur Re-Formatierung nach dem Kalendermaß genutzt wird. Nicht zufällig sind in beiden Kalendern die epochalen Ereignisse der Schreib-Gegenwart mit dezidierten Schreibprozessen des Erinnerns und Vergegenwärtigens verbunden, in einem kleinen „Ensemble von Sprache, Instrumentalität und Geste“, wie Campe es beschrieben hat. Doch offensichtlich hat Goethe dieses wiederum geteilt, mindestens mit den identifizierten Mit-Schreibenden Stadelmann und Graf Piotrowski in der kollektiven Praktik des Schreibens, die es offenbar auch nahelegte oder erforderte, die im engeren Sinn privaten Notizen zu codieren, wie das Beispiel der Tabelle gezeigt hat.

Die im selben Kalender von 1823 skizzierten Verse für Ulrike von Levetzow haben dagegen offensichtlich nicht diesen Charakter, da sie einerseits für Billetts konzipiert sind und mithin für ein kollektiv gelesenes Medium der galanten Kommunikation unter aller Augen. Andererseits belegt die teils geradezu kalligraphische Gestaltung spruchartiger Verse mit Tinte wie deren Ergänzung durch die französische Äußerung des Grafen von dessen Hand, dass der Kalender zumindest an diesen Stellen nicht nur wie ein Stammbuch genutzt, sondern offenbar entsprechend auch fremden Blicken geöffnet wurde - einschließlich seiner pragmatischen Nutzung für Notizen zu den

69 „Durch die Zusammenstellung zur Trilogie rückt nun die ,Elegie‘ in ein neues Licht. An sich ist sie ein sehr stilisiertes Gedicht, das ein ganz allgemeines Ich schildert. Da aber im Gedicht ,An Werther' das Goethesche Ich sehr persönlich spricht, rückt nun auch die Elegie in diesen individuellen Zusammenhang“ (Trunz, „Goethes späte Lyrik“ [Anm. 54], S. 155). 
Maximen und Reflexionen und einen Briefentwurf zur weiteren Bearbeitung auch durch die Schreiber, die am Ende der beschriebenen Seiten stehen. ${ }^{70}$ Sie könnten gut auch noch von einer jener Listen gefolgt werden, wie sie Stadelmann zur Haushaltsführung im Kalender 1822 angelegt hatte. ${ }^{71}$ Der Kalender, der sich ohnehin als Medium der Selbstvergewisserung, der Bilanz, der Vorund Rückschau und des Abrechnens anbietet und für diese Nutzung auch entsprechende Vorgaben macht, ermöglicht so in seiner Verdopplung auf der Böhmen-Reise von 1823 zudem auf jeder Ebene kollektives Schreiben und das Schreiben vom Kollektiv, sei es des Geschriebenen oder der Schreibenden. Als Aktant mit eigenen Angeboten zur Öffnung und Schließung von Schreibräumen ist er in seiner überlieferten Form jedoch um das entscheidende Element vermindert, das überhaupt erst die Bedingung der Möglichkeit für alles Weitere darstellt: Beiden Taschenkalendern fehlt heute die kleine Stoffschlaufe, die einmal den entscheidenden zweiten Aktant gefasst hat, einen kleinen Bleistift, immer zur Hand, für das Schreiben in jeder Lebenslage. Ihn hat Goethe in einer metonymischen Gleichsetzung von Schreibendem und Schreibwerkzeug in der eingangs zitierten autobiographischen Schreibszene emphatisch beschrieben, weil er „williger die Züge hergab“. ${ }^{72}$ Wie Martin Stingelin bemerkt, „[steht hier] der Eigenwille des Schreibwerkzeugs also in Form der Feder der größeren ,Willigkeit' in Form des Bleistifts gegenüber “. ${ }^{\prime 7}$ Welchen Anteil dieses neue Werkzeug an der Erprobung von Schreib-Formen gehabt hat, demonstrieren die beiden Kalender mit ihren Verweisen auf die Billetts, Gaben und Gedichte für Ulrike von Levetzow besonders eindrucksvoll, weil sie buchstäblich das Nebeneinander von offener gemeinsamer Schreibarbeit mit Stadelmann, dem Grafen sowie (potentiell) weiteren Schreibenden und der chiffrierten Antizipation einer anderen Gemeinschaft mit der adressierten fernen Geliebten im Schreib-Ensemble anzeigen. So finden sich Kollektive aller Art gerade dort, wo gemeinhin Urszenen einsamen Schreibens vermutet werden würden.

$70 \quad 1823$ waren neben Stadelmann auch Johann August Friedrich John und Michael Färber für Goethe als Schreiber tätig; auf der Böhmen-Reise 1823 waren Stadelmann und John mit vielfältigen Aufgaben betraut.

71 Behrens/Michel, „Anhang“ (Anm. 35), S. 147f.

72 Johann Wolfgang Goethe, Aus meinem Leben. Dichtung und Wahrheit, in: Goethes Werke (Anm. 10), Bd. 10, S. 81.

73 Stingelin, ,,Schreiben“ (Anm. 6), S. 9. 